\title{
Sets of nondifferentiability for conjugacies between expanding interval maps
}

\author{
by \\ Thomas Jordan (Bristol), Marc Kesseböhmer (Bremen), \\ Mark Pollicott (Coventry) and Bernd O. Stratmann (St Andrews)
}

Dedicated to Michat Misiurewicz on the occasion of his 60 th birthday

\begin{abstract}
We study differentiability of topological conjugacies between expanding piecewise $C^{1+\epsilon}$ interval maps. If these conjugacies are not $C^{1}$, then their derivative vanishes Lebesgue almost everywhere. We show that in this case the Hausdorff dimension of the set of points for which the derivative of the conjugacy does not exist lies strictly between zero and one. Moreover, by employing the thermodynamic formalism, we show that this Hausdorff dimension can be determined explicitly in terms of the Lyapunov spectrum. These results then give rise to a "rigidity dichotomy" for the type of conjugacies under consideration.
\end{abstract}

1. Introduction and statement of results. In this paper we study aspects of nondifferentiability for conjugacy maps between expanding maps of the unit interval $\mathcal{U}$ into itself which have precisely $d$ increasing full inverse branches and each of these branches is a strictly contracting $C^{1+\epsilon}$ diffeomorphism on $\mathcal{U}$, for some fixed $\epsilon>0$ and some fixed integer $d \geq 2$ (here, a map $f: \mathcal{U} \rightarrow f(\mathcal{U}) \subset \mathbb{R}$ is said to be a $C^{1+\epsilon}$ diffeomorphism if there exists an extension $\tilde{f}$ of $f$ to some open neighbourhood of $\mathcal{U}$ which is a diffeomorphism such that $\tilde{f}^{\prime} \mid \mathcal{U}$ is Hölder continuous with Hölder exponent equal to $\epsilon$ ). Throughout, these expanding maps will be referred to as expanding piecewise $C^{1+\epsilon}$ maps. Clearly, each map of this type is naturally semi-conjugate to the full shift $\Sigma$ over the alphabet $A:=\{1, \ldots, d\}$. Moreover, if $S$ and $T$ are two expanding piecewise $C^{1+\epsilon}$ maps, then the following diagram commutes, where $\sigma$ refers to the usual shift map on $\Sigma$, and $\pi_{S}$ and $\pi_{T}$ denote the associated coding

2010 Mathematics Subject Classification: Primary 37C45; Secondary 28A80, 37A10. Key words and phrases: interval maps, conjugacies, rigidity, sets of nondifferentiability. 
maps:

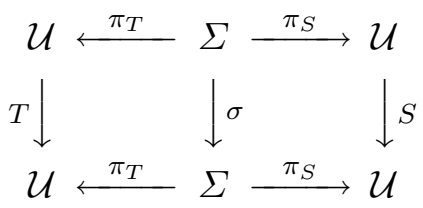

The conjugacy map $\Theta: \mathcal{U} \rightarrow \mathcal{U}$ between the two systems $(\mathcal{U}, S)$ and $(\mathcal{U}, T)$ is then given by $T \circ \Theta=\Theta \circ S$ (see Figs. 1 and 4 for some examples), and one immediately verifies that $\Theta$ is continuous. The first main task of the paper will be to employ the thermodynamic formalism in order to give a detailed fractal analysis of the following three sets:

$$
\begin{aligned}
\mathcal{D}_{\sim} & =\mathcal{D}_{\sim}(S, T):=\left\{\xi \in \mathcal{U}: \Theta^{\prime}(\xi) \text { does not exist in the generalised sense }\right\} \\
\mathcal{D}_{\infty} & =\mathcal{D}_{\infty}(S, T):=\left\{\xi \in \mathcal{U}: \Theta^{\prime}(\xi)=\infty\right\} \\
\mathcal{D}_{0} & =\mathcal{D}_{0}(S, T):=\left\{\xi \in \mathcal{U}: \Theta^{\prime}(\xi)=0\right\} .
\end{aligned}
$$

Here, $\Theta^{\prime}(\xi)$ exists in the generalised sense means that $\Theta^{\prime}(\xi)$ either exists or else is equal to infinity (at the boundary points we interpret these quantities in terms of limits from the left or right, as appropriate). Note that we can trivially write $\mathcal{U}=\mathcal{D} \sim \cup \mathcal{D}_{\infty} \cup \mathcal{D}_{0} \cup \mathcal{D}$ where $\mathcal{D}:=\left\{\xi \in \mathcal{U}: \Theta^{\prime}(\xi) \in(0, \infty)\right\}$. However, as we will see, either $\mathcal{D}=\mathcal{U}$ or $\mathcal{D}=\emptyset$.

The second main task of this paper will be to find a necessary and sufficient condition for two expanding piecewise $C^{1+\epsilon}$ systems to be rigid in a certain sense.

To state our main results in greater detail, we first need to recall some concepts from the thermodynamic formalism. Let $\varphi, \psi: \Sigma \rightarrow \mathbb{R}_{<0}$ denote the Hölder continuous potential functions which are given for $x=$ $\left(x_{1} x_{2} \ldots\right) \in \Sigma$ by

$$
\varphi(x):=\log \left(S_{x_{1}}^{-1}\right)^{\prime}\left(\pi_{S}(\sigma(x))\right) \quad \text { and } \quad \psi(x):=\log \left(T_{x_{1}}^{-1}\right)^{\prime}\left(\pi_{T}(\sigma(x))\right),
$$

where $S_{a}^{-1}$ and $T_{a}^{-1}$ denote the inverse branches of $S$ and $T$ associated with $a \in A$. Also, let $P$ denote the usual pressure function from the thermodynamic formalism (see [17] for the definition of $P$ ). Then there exists a function $\beta: \mathbb{R} \rightarrow \mathbb{R}$ given implicitly by the pressure equation

$$
P(s \varphi+\beta(s) \psi)=0 \quad \text { for all } s \in \mathbb{R} .
$$

Note that $\beta$ is well defined, since $\psi<0$. With $\mu_{s}$ denoting the equilibrium measure associated with the potential function $s \varphi+\beta(s) \psi$, we have

$$
\beta^{\prime}(s):=\frac{-\int \varphi d \mu_{s}}{\int \psi d \mu_{s}}<0 .
$$

Therefore, $\beta$ is strictly decreasing, and one also immediately verifies that $\beta(1)=0$ and $\beta(0)=1$. If $\varphi$ and $\psi$ are cohomologically independent, that is, if there are no nontrivial choices of $b, c \in \mathbb{R}$ and $u \in C(\Sigma)$ such that 
$b \varphi+c \psi=u \circ \sigma-u$ (in this situation, we will also say that $S$ and $T$ are cohomologically independent), then $\beta$ is strictly convex (see e.g. [17]). In this situation the mean value theorem for derivatives gives that there exists a unique $s_{0} \in(0,1)$ such that $\beta^{\prime}\left(s_{0}\right)=-1$. Let $\widetilde{\beta}: \mathbb{R} \rightarrow \mathbb{R}$ be defined by $\widetilde{\beta}(s):=\beta(s)+s$. Note that $\widetilde{\beta}$ is convex and has a unique minimum at $s_{0}$. Also, $\widetilde{\beta}(0)=\widetilde{\beta}(1)=1$ and $\widetilde{\beta}\left(s_{0}\right)=\widehat{\beta}(1)$, where $\widehat{\beta}$ denotes the (concave) Legendre transform of $\beta$, given by $\widehat{\beta}(s):=\inf _{t \in \mathbb{R}}(\beta(t)+s t)$ for $s \in \mathbb{R}$. Finally, the level sets $\mathcal{L}(s)$ are defined by

$$
\mathcal{L}(s):=\left\{\xi \in \mathcal{U}: \lim _{n \rightarrow \infty} \frac{\mathcal{S}_{n} \varphi\left(\pi_{S}^{-1}(\xi)\right)}{\mathcal{S}_{n} \psi\left(\pi_{T}^{-1}(\xi)\right)}=s\right\},
$$

where $\mathcal{S}_{n} f:=\sum_{k=0}^{n-1} f \circ \sigma^{k}$ denotes the $n$th Birkhoff sum. By standard thermodynamic formalism (see e.g. [17]), we then have, for $s$ in the closure $\overline{-\beta^{\prime}(\mathbb{R})}$ of the range of $-\beta^{\prime}$,

$$
\operatorname{dim}_{H}(\mathcal{L}(s))=\widehat{\beta}(s) / s=\frac{1}{s} \inf _{t \in \mathbb{R}}(s t+\beta(t))=\inf _{t \in \mathbb{R}}(t+\beta(t) / s),
$$

whereas for $s \notin \overline{-\beta^{\prime}(\mathbb{R})}$ we have $\mathcal{L}(s)=\emptyset$.

The first main result of this paper is now stated in the following theorem.

Theorem 1.1. Let $S$ and $T$ be two cohomologically independent expanding piecewise $C^{1+\epsilon}$ maps. Then

$$
0<\operatorname{dim}_{\mathrm{H}}\left(\mathcal{D}_{\sim}\right)=\operatorname{dim}_{\mathrm{H}}\left(\mathcal{D}_{\infty}\right)=\operatorname{dim}_{\mathrm{H}}(\mathcal{L}(1))=\widetilde{\beta}\left(s_{0}\right)<1 .
$$

Using the well known fact that both $T$ and $S$ have absolutely continuous invariant measures with $C^{\epsilon}$ densities (see e.g. [1, Theorem 2.1]), one immediately verifies that either $\Theta^{\prime}=0$ Lebesgue almost everywhere or $\Theta$ is $C^{1+\epsilon}$. The second main result of this paper is to give a refinement of this dichotomy, and this is summarized in the following theorem.

TheOREM 1.2. Let $S$ and $T$ be two expanding piecewise $C^{1+\epsilon}$ maps. Then

the conjugacy $\Theta$ is a $C^{1+\epsilon}$ diffeomorphism $\Leftrightarrow \operatorname{dim}_{\mathrm{H}}\left(\mathcal{D}_{\sim}\right)=0$.

More precisely, the following "rigidity dichotomy" holds.

(1) If $S$ and $T$ are cohomologically dependent, then $\Theta$ is a $C^{1+\epsilon}$ diffeomorphism and hence absolutely continuous. Equivalently,

$$
\mathcal{D}_{0}=\mathcal{D}_{\infty}=\mathcal{D}_{\sim}=\emptyset, \quad \text { and hence } \mathcal{U}=\left\{\xi \in \mathcal{U}: 0<\Theta^{\prime}(\xi)<\infty\right\} .
$$

(2) If $S$ and $T$ are cohomologically independent, then $\Theta$ is singular, that is, $\lambda\left(\mathcal{D}_{0}\right)=1$. Moreover, $\Theta$ is Hölder continuous with Hölder exponent equal to $\left(\sup _{x \in \mathbb{R}}-\beta^{\prime}(x)\right)^{-1}$, and

$$
0<\operatorname{dim}_{\mathrm{H}}\left(\mathcal{D}_{\infty}\right)=\operatorname{dim}_{\mathrm{H}}\left(\mathcal{D}_{\sim}\right)<1 .
$$


The latter theorem is closely related to classical work by Shub and Sullivan [21] on the smoothness of conjugacies between expanding maps of the unit circle $\mathbb{S}^{1}$ (see also e.g. $[2,9,16,22]$ ). In [21] it was shown that if the conjugacy between two $C^{k}$ expanding maps is absolutely continuous, for some $k \geq 2$, then the conjugacy is necessarily $C^{k}$ too (also cf. [18]). Let us also mention a result by Cui [3] which states that the conjugacy map between two expanding $C^{1+\epsilon}$ circle endomorphisms is itself $C^{1+\epsilon}$, if it has finite, nonzero derivative at some point in $\mathbb{S}^{1}$. So, to deduce Theorem 1.2 from Theorem 1.1, we need to adapt this result to the setting of interval maps.

In the case of circle maps we can combine our result on interval maps and the result of Cui to obtain a result for endomorphisms of $\mathbb{S}^{1}$. For this one has to rewrite Theorem 1.1 in terms of orientation preserving expanding $C^{1+\epsilon}$ circle maps, and this then gives rise to the following result.

Corollary 1.3. For the conjugacy map $\Phi$ between a given pair $\left(\mathbb{S}^{1}, U\right)$ and $\left(\mathbb{S}^{1}, V\right)$ of expanding $C^{1+\epsilon}$ endomorphisms of $\mathbb{S}^{1}$, the following statements are equivalent.

(1) $\Phi$ is a $C^{1+\epsilon}$ circle map;

(2) $\operatorname{dim}_{\mathrm{H}}\left(\left\{\xi \in \mathbb{S}^{1}: \Phi^{\prime}(\xi)\right.\right.$ does not exist in the generalised sense $\left.\}\right)=0$;

(3) $\operatorname{dim}_{\mathrm{H}}\left(\left\{\xi \in \mathbb{S}^{1}: 0<\Phi^{\prime}(\xi)<\infty\right\}\right)=1$;

(4) $\Phi$ is absolutely continuous;

(5) $\Phi$ is bi-Lipschitz.

A natural question to ask is how the quantities $\operatorname{dim}_{H}\left(\mathcal{D}_{\infty}(S, T)\right)$ and $\operatorname{dim}_{\mathrm{H}}\left(\mathcal{D}_{\sim}(S, T)\right)$ vary as $S$ and $T$ change. The next two results address this question.

Proposition 1.4. For a $C^{k}$ family of expanding maps, the Hausdorff dimension of the set of nondifferentiability points has a $C^{k-2}$ dependence.

Proposition 1.5. There exists a pair of $C^{2}$ endomorphisms of $\mathbb{S}^{1}$ for which the set of nondifferentiability points for the associated conjugacy map has arbitrarily small Hausdorff dimension.

The paper is organised as follows. In Sections 2 and 3 we give the proofs of Theorems 1.1 and 1.2. Section 4 discusses two basic examples, and one of these is then used in Section 5 for the proof of Proposition 1.5. In Section 5 we also give the proof of Proposition 1.4.

REMARK 1.6. (1) Note that

$$
\mathcal{D}_{\sim}(S, T) \cup \mathcal{D}_{\infty}(S, T)=\{\xi \in \mathcal{U}: \Theta \text { is not differentiable at } \xi\},
$$

and hence Theorem 1.1 in particular implies that if $S$ and $T$ are cohomologically independent, then the Hausdorff dimension of the set of points for which $\Theta$ is not differentiable is equal to $\widetilde{\beta}\left(s_{0}\right)$. 
(2) There is also a variational formula for the Hausdorff dimension of $\mathcal{D}_{\sim}$. Namely, as we will see in Section 2.3,

$$
\operatorname{dim}_{\mathrm{H}}\left(\mathcal{D}_{\sim}\right)=\sup \left\{\frac{h(\mu)}{\int \varphi d \mu}: \frac{\int \varphi d \mu}{\int \psi d \mu}=1\right\}
$$

where the supremum ranges over all $\sigma$-invariant probability measures on $\Sigma$. In particular, this variational formula shows that if we swap the roles of $\varphi$ and $\psi$, then this has no effect on the Hausdorff dimension of the set of nondifferentiability. In other words, if instead of $\Theta$ we take the dual conjugacy $\widehat{\Theta}$, given by $S \circ \widehat{\Theta}=\widehat{\Theta} \circ T$, then the Hausdorff dimension of the set of points at which $\widehat{\Theta}^{\prime}$ does not exist in the generalised sense coincides with $\operatorname{dim}_{H}\left(\mathcal{D}_{\sim}\right)$, i.e.

$$
\operatorname{dim}_{\mathrm{H}}\left(\mathcal{D}_{\sim}(S, T)\right)=\operatorname{dim}_{\mathrm{H}}\left(\mathcal{D}_{\sim}(T, S)\right) .
$$

(3) The conjugacy map $\Theta$ can also be viewed as the distribution function of the measure $m_{\Theta}:=\lambda \circ \Theta$. This follows, since for $\xi \in \mathcal{U}$ we have

$$
m_{\Theta}([0, \xi))=\lambda([0, \Theta(\xi)))=\Theta(\xi) .
$$

Hence, the investigations in this paper can also be seen as a study of singular distribution functions with support equal to $\mathcal{U}$. Note that there are strong parallels to the results in [12], where we used some of the outcomes of [13] to give a fractal analysis of nondifferentiability for Minkowski's question mark function.

(4) Finally, let us mention that the statements in Theorems 1.1 and 1.2 can be generalised so that the derivative of $\Theta$ gets replaced by the $s$-Hölder derivative $\Delta_{s} \Theta$ of $\Theta$, given for $s \in-\beta^{\prime}(\mathbb{R})$ by

$$
\left(\Delta_{s} \Theta\right)(\xi):=\lim _{\eta \rightarrow \xi} \frac{|\Theta(\eta)-\Theta(\xi)|}{|\eta-\xi|^{s}} .
$$

For this more general derivative the relevant sets are $\mathcal{D}_{\sim}^{(s)}=\mathcal{D}_{\sim}^{(s)}(S, T):=\left\{\xi \in \mathcal{U}:\left(\Delta_{s} \Theta\right)(\xi)\right.$ does not exist $\mathcal{D}_{\infty}^{(s)}=\mathcal{D}_{\infty}^{(s)}(S, T):=\left\{\xi \in \mathcal{U}:\left(\Delta_{s} \Theta\right)(\xi)=\infty\right\}$. in the generalised sense\}, Straightforward adaptations of the proofs in this paper then show that

$$
\operatorname{dim}_{\mathrm{H}}\left(\mathcal{D}_{\sim}^{(s)}\right)=\operatorname{dim}_{\mathrm{H}}\left(\mathcal{D}_{\infty}^{(s)}\right)=\operatorname{dim}_{\mathrm{H}}(\mathcal{L}(s)) .
$$

In particular, this shows that on $-\beta^{\prime}(\mathbb{R})$ the Lyapunov spectrum $s \mapsto \widehat{\beta}(s) / s$ coincides with the "spectrum of non-s-Hölder differentiability of $\Theta$ ". Note that, by generalising results of $[3,7]$ and others, in [11] similar results were obtained for certain classes of Cantor-like sets. 


\section{Proof of Theorem 1.1}

2.1. The geometry of the derivative of $\Theta$. Let us first introduce some notations which will be used throughout.

Definition. Let us say that $x=\left(x_{1} x_{2} \ldots\right) \in \Sigma$ has an $i$-block of length $k$ at the $n$th level, for $n, k \in \mathbb{N}$ and $i \in\{1, d\}$, if $x_{n+k+1} \in A \backslash\{i\}$ and $x_{n+m}=i$ for all $m \in\{1, \ldots, k\}$. Moreover, we will say that $x=\left(x_{1} x_{2} \ldots\right) \in \Sigma$ has a strict $i$-block of length $k$ at the $n$th level if additionally $x_{n} \in A \backslash\{i\}$.

For ease of exposition, we define the function $\chi: \Sigma \rightarrow \mathbb{R}$ by $\chi:=\psi-\varphi$. Also, let $D_{\Theta}(\xi, \eta)$ denote the differential quotient for $\Theta$ at $\xi$ and $\eta$, that is,

$$
D_{\Theta}(\xi, \eta):=\frac{\Theta(\xi)-\Theta(\eta)}{\xi-\eta} .
$$

Moreover, we use $\underline{a}_{k}$ to denote the word of length $k \in \mathbb{N}$ containing exclusively the letter $a \in A$, and we let $\underline{a}$ denote the infinite word containing exclusively the letter $a \in A$. Also, $\left[x_{1} \ldots x_{n}\right]$ denotes the cylinder set associated with the finite word $\left(x_{1}, \ldots, x_{n}\right) \in A^{n}$, that is,

$$
\left[x_{1} \ldots x_{n}\right]:=\left\{\left(y_{1} y_{2} \ldots\right) \in \Sigma: y_{i}=x_{i} \text { for all } i=1, \ldots, n\right\} .
$$

Throughout, " $\simeq$ " means that the ratio of the left hand side to the right hand side is uniformly bounded away from zero and infinity. Likewise, we use "«" to denote that the expression on the left hand side is uniformly bounded by the expression on the right hand side multiplied by some fixed positive constant.

Let us begin our discussion of the geometry of the derivative of $\Theta$ with the following crucial geometric observation.

Proposition 2.1. Let $x=\left(x_{1} x_{2} \ldots\right), y=\left(y_{1} y_{2} \ldots\right) \in \Sigma$ satisfy $y \in$ $\left[x_{1} \ldots x_{n-1}\right]$ as well as $x_{n}=a$ and $y_{n}=b$ for some $n \in \mathbb{N}$ and $a, b \in A$ with $|a-b|=1$ (note that for $n=1$ we adopt the convention that $x_{1}=a$ and $\left.y_{1}=b\right)$. Moreover, assume that for some $k, l \in \mathbb{N}$, the word $x$ has an $i$-block of length $k$ at the $n$th level, and $y$ has a $j$-block of length $l$ at the $n$th level. Here, $i, j \in\{1, d\}$ are chosen such that if $a<b$ then $i=d$ and $j=1$, whereas if $a>b$ then $i=1$ and $j=d$. In this situation for $\xi:=\pi_{S}(x)$ and $\eta:=\pi_{S}(y)$

$$
D_{\Theta}(\xi, \eta) \asymp e^{\mathcal{S}_{n} \chi(x)} \frac{e^{k \psi((\underline{i}))}+e^{l \psi((\underline{j}))}}{e^{k \varphi((\underline{i}))}+e^{l \varphi((\underline{j}))}} .
$$

Proof. We only consider the case $a=b+1>b$. The case $a<b$ is completely analogous and is left to the reader. In this situation, for some $p \in A \backslash\{1\}$ and $q \in A \backslash\{d\}$, the words $x$ and $y$ are of the form

$$
x=\left(x_{1} \ldots x_{n-1} a \underline{1}_{k} p \ldots\right) \text { and } y=\left(x_{1} \ldots x_{n-1} b \underline{d}_{l} q \ldots\right) .
$$


Then consider the following cylinder sets:

$$
\begin{array}{ll}
I_{1}:=\pi_{S}\left(\left[x_{1} \ldots x_{n-1} b \underline{d}_{l+1}\right]\right) & I_{2}:=\pi_{S}\left(\left[x_{1} \ldots x_{n-1} a \underline{1}_{k+1}\right]\right), \\
J_{1}:=\pi_{S}\left(\left[x_{1} \ldots x_{n-1} b \underline{d}_{l}\right]\right) & J_{2}:=\pi_{S}\left(\left[x_{1} \ldots x_{n-1} a \underline{1}_{k}\right]\right) .
\end{array}
$$

One immediately verifies that

$$
I_{1} \cup I_{2} \subset[\eta, \xi] \subset J_{1} \cup J_{2} .
$$

Moreover, with $\eta^{\prime}:=\pi_{T}\left(\left(x_{1} \ldots x_{n-1} b \underline{d}\right)\right)=\pi_{T}\left(\left(x_{1} \ldots x_{n-1} a \underline{1}\right)\right)$ we have, using the bounded distortion property,

$|\Theta(\xi)-\Theta(\eta)|=\left|\Theta(\xi)-\Theta\left(\eta^{\prime}\right)\right|+\left|\Theta\left(\eta^{\prime}\right)-\Theta(\eta)\right| \asymp e^{\mathcal{S}_{n} \psi(x)}\left(e^{k \psi((\underline{d}))}+e^{l \psi((\underline{1}))}\right)$.

Similarly, one obtains

$$
\begin{aligned}
|\xi-\eta| \asymp \operatorname{diam}\left(I_{1}\right)+\operatorname{diam}\left(I_{2}\right) & \asymp \operatorname{diam}\left(J_{1}\right)+\operatorname{diam}\left(J_{2}\right) \\
& \asymp e^{\mathcal{S}_{n} \varphi(x)}\left(e^{k \varphi((\underline{d}))}+e^{l \varphi((\underline{1}))}\right) .
\end{aligned}
$$

Note that Proposition 2.1 does in particular contain all the cases in which, for given $x, y \in \Sigma, D_{\Theta}\left(\pi_{S}(x), \pi_{S}(y)\right)$ can significantly deviate from $\exp \left(\mathcal{S}_{n} \chi(x)\right)$. This is clarified by the following lemma, which addresses the cases not covered by Proposition 2.1.

Lemma 2.2. Let $x=\left(x_{1} x_{2} \ldots\right), y=\left(y_{1} y_{2} \ldots\right) \in \Sigma$ be such that $y \in$ $\left[x_{1} \ldots x_{n-1}\right] \backslash\left[x_{1} \ldots x_{n}\right]$ and either $\left|x_{n}-y_{n}\right|>1$, or if $\left|x_{n}-y_{n}\right|=1$ then $\pi_{S}\left(\left[x_{1} \ldots x_{n+1}\right]\right) \cap \pi_{S}\left(\left[y_{1} \ldots y_{n+1}\right]\right)=\emptyset$. For $\xi:=\pi_{S}(x)$ and $\eta:=\pi_{S}(y)$,

$$
D_{\Theta}(\xi, \eta) \asymp e^{\mathcal{S}_{n} \chi(x)} .
$$

Proof. We have either $\pi_{S}\left(\left[x_{1} \ldots x_{n}\right]\right) \cap \pi_{S}\left(\left[y_{1} \ldots y_{n}\right]\right)=\emptyset$, and hence there exists an interval separating these two sets, or if $\pi_{S}\left(\left[x_{1} \ldots x_{n}\right]\right) \cap$ $\pi_{S}\left(\left[y_{1} \ldots y_{n}\right]\right) \neq \emptyset$ then $\pi_{S}\left(\left[x_{1} \ldots x_{n+1}\right]\right) \cap \pi_{S}\left(\left[y_{1} \ldots y_{n+1}\right]\right)=\emptyset$. Clearly, in both cases there exist $a, b \in A$ such that the interval $I_{a b}:=\pi_{S}\left(\left[x_{1} \ldots x_{n-1} a b\right]\right)$ separates the intervals $\pi_{S}\left(\left[x_{1} \ldots x_{n+1}\right]\right)$ and $\pi_{S}\left(\left[y_{1} \ldots y_{n+1}\right]\right)$. Using this, we then obtain

$$
\begin{aligned}
e^{\mathcal{S}_{n} \psi(x)} & \ll \operatorname{diam}\left(\Theta\left(I_{a b}\right)\right) \ll|\Theta(\xi)-\Theta(\eta)| \\
& \ll \operatorname{diam}\left(\Theta\left(\pi_{S}\left(\left[x_{1} \ldots x_{n-1}\right]\right)\right)\right) \ll e^{\mathcal{S}_{n} \psi(x)},
\end{aligned}
$$

and

$$
e^{\mathcal{S}_{n} \varphi(x)} \ll \operatorname{diam}\left(I_{a b}\right) \ll|\xi-\eta| \ll \operatorname{diam}\left(\pi_{s}\left(\left[x_{1} \ldots x_{n-1}\right]\right)\right) \ll e^{\mathcal{S}_{n} \varphi(x)} .
$$

Lemma 2.3. If $x=\left(x_{1} x_{2} \ldots\right) \in \Sigma$ has an $i$-block of length $k$ at the $n$th level, for some $n, k \in \mathbb{N}$ and $i \in\{1, d\}$, then for each $\eta \in \pi_{s}\left(\left[x_{1} \ldots x_{n}\right] \backslash\right.$ $\left.\left[x_{1} \ldots x_{n+1}\right]\right)$, with $\delta:=-\min \{\psi((\underline{1})), \psi((\underline{d}))\}>0$ and $\xi:=\pi_{S}(x)$,

$$
D_{\Theta}(\xi, \eta) \gg e^{\mathcal{S}_{n} \chi(x)} e^{-\delta k} \text {. }
$$


Proof. Trivially, $|\xi-\eta| \ll \exp \left(\mathcal{S}_{n} \varphi(x)\right)$. As in the proof of the previous lemma, one immediately verifies that

$$
|\Theta(\xi)-\Theta(\eta)| \gg e^{\mathcal{S}_{n+k} \psi(x)} \gg e^{\mathcal{S}_{n} \psi(x)} e^{-\delta k} .
$$

By combining these observations, the result follows.

Lemma 2.4. Let $x \in \Sigma$ and $\xi:=\pi_{S}(x)$.

(1) If $\lim \sup _{n \rightarrow \infty} e^{\mathcal{S}_{n} \chi(x)}=\infty$, then $\lim \sup _{\eta \rightarrow \xi} D_{\Theta}(\xi, \eta)=\infty$.

(2) If $\liminf _{n \rightarrow \infty} e^{\mathcal{S}_{n} \chi(x)}=0$, then $\liminf _{\eta \rightarrow \xi} D_{\Theta}(\xi, \eta)=0$.

Proof. Let $x=\left(x_{1} x_{2} \ldots\right)$ and assume without loss of generality that

$$
\xi \notin \pi_{S}\left(\left\{\left(x_{1} x_{2} \ldots\right) \in \Sigma: \exists n \in \mathbb{N} \exists i \in\{1, d\} \forall k \geq n: x_{k}=i\right\}\right) .
$$

For $n \in \mathbb{N}$, the left and right boundary points of $\pi_{S}\left(\left[x_{1} \ldots x_{n}\right]\right)$ are given by $\xi_{n}:=\pi_{S}\left(\left(x_{1} \ldots x_{n} \underline{1}\right)\right)$ and $\eta_{n}:=\pi_{S}\left(\left(x_{1} \ldots x_{n} \underline{d}\right)\right)$. By assumption we have $\xi \notin\left\{\xi_{n}, \eta_{n}: n \in \mathbb{N}\right\}$. It then follows that

$$
\min \left\{D_{\Theta}\left(\xi, \eta_{n}\right), D_{\Theta}\left(\xi, \xi_{n}\right)\right\} \leq D_{\Theta}\left(\xi_{n}, \eta_{n}\right) \leq \max \left\{D_{\Theta}\left(\xi, \eta_{n}\right), D_{\Theta}\left(\xi, \xi_{n}\right)\right\} .
$$

Since $D_{\Theta}\left(\xi_{n}, \eta_{n}\right) \asymp e^{\mathcal{S}_{n} \chi(\xi)}$, the lemma follows.

We have the following immediate corollary.

Corollary 2.5. Let $x \in \Sigma$ be such that

$$
\liminf _{n \rightarrow \infty} e^{\mathcal{S}_{n} \chi(x)}=0 \quad \text { and } \quad \limsup _{n \rightarrow \infty} e^{\mathcal{S}_{n} \chi(x)}=\infty .
$$

Then $\pi_{S}(x) \in \mathcal{D}_{\sim}$.

For the remainder of this section we restrict the discussion to the following two cases:

$$
\begin{array}{ll}
\text { CASE 1: } & \psi((\underline{1})) / \varphi((\underline{1}))<\min \{\psi((\underline{d})) / \varphi((\underline{d})), 1\} ; \\
\text { CASE 2: } & \psi((\underline{d})) / \varphi((\underline{d}))<\min \{\psi((\underline{1})) / \varphi((\underline{1})), 1\} .
\end{array}
$$

As we will see in Lemma 2.7, these two cases are in fact the only relevant cases for the purposes in this paper. In fact, without loss of generality, we will always assume that we are in the situation of Case 1. The discussion of Case 2 is completely analogous (essentially, one has to interchange the roles of 1 and $d$ as well as of $l$ and $k$ ), and will be left to the reader. Note that Cases 1 and 2 include the cases

$$
e^{\chi((\underline{1}))}>1>e^{\chi((\underline{d}))} \text { and } e^{\chi((\underline{1}))}<1<e^{\chi((\underline{d}))},
$$

which are for instance fulfilled in the Salem examples (Example 1) briefly discussed in Section 4.

On the basis of this assumption, we now make the following crucial observation. 
Lemma 2.6. Assume that we are in Case 1 of (2.1). Then for all $l \in \mathbb{N}$,

$$
\frac{e^{l \psi((\underline{1}))}+e^{k \psi((\underline{d}))}}{e^{l \varphi((\underline{1}))}+e^{k \varphi((\underline{d}))}} \ll e^{\alpha k},
$$

where $\alpha:=\chi((\underline{1})) \varphi((\underline{d})) / \varphi((\underline{1}))>0$. Moreover, if $l=\lfloor k \alpha / \chi((\underline{1}))\rfloor$ then

$$
\frac{e^{l \psi((\underline{1}))}+e^{k \psi((\underline{d}))}}{e^{l \varphi((\underline{1}))}+e^{k \varphi((\underline{d}))}} \gg e^{\alpha k}
$$

Here, $\lfloor r\rfloor$ denotes the smallest integer greater than or equal to $r \in \mathbb{R}$.

Proof. First note that with $\alpha^{\prime}:=\varphi((\underline{d})) / \varphi((\underline{1}))$ the conditions in Case 1 immediately imply

$$
e^{\varphi((\underline{1}))}<e^{\psi((\underline{1}))} \quad \text { and } \quad e^{\psi((\underline{d}))}<e^{\alpha^{\prime} \psi((\underline{1}))} .
$$

In particular, this implies that $\chi((\underline{1}))>0$. Then for all $l \geq \alpha^{\prime} k$,

$$
\frac{e^{l \psi((\underline{1}))}+e^{k \psi((\underline{d}))}}{e^{l \varphi((\underline{1}))}+e^{k \varphi((\underline{d}))}} \leq \frac{e^{l \psi((\underline{1}))}+e^{k \alpha^{\prime} \psi((\underline{1}))}}{e^{k \varphi((\underline{d}))}} \leq 2 \frac{e^{k \alpha^{\prime} \psi((\underline{1}))}}{e^{k \varphi((\underline{d}))}}=2 \frac{e^{k \alpha^{\prime} \psi((\underline{1}))}}{e^{k \alpha^{\prime} \varphi((\underline{1}))}}=2 e^{\alpha k} .
$$

If $l \leq \alpha^{\prime} k$, then we obtain

$$
\begin{aligned}
\frac{e^{l \psi((\underline{1}))}+e^{k \psi((\underline{d}))}}{e^{l \varphi((\underline{1}))}+e^{k \varphi((\underline{d}))}} & \leq \frac{e^{l \psi((\underline{1}))}+e^{k \alpha^{\prime} \psi((\underline{1}))}}{e^{l \varphi((\underline{1}))}} \\
& \leq 2\left(\frac{e^{\psi((\underline{1}))}}{e^{\varphi((\underline{1}))}}\right)^{l} \leq 2\left(\frac{e^{\psi((\underline{1}))}}{e^{\varphi((\underline{1}))}}\right)^{\alpha^{\prime} k}=2 e^{\alpha k} .
\end{aligned}
$$

Finally, if $l=\left\lfloor\alpha^{\prime} k\right\rfloor$ then

$$
\frac{e^{l \psi((\underline{1}))}+e^{k \psi((\underline{d}))}}{e^{l \varphi((\underline{1}))}+e^{k \varphi((\underline{d}))}} \asymp \frac{e^{l \psi((\underline{1}))}+e^{k \psi((\underline{d}))}}{2 e^{k \varphi((\underline{d}))}} \gg \frac{e^{k \alpha^{\prime} \psi((\underline{1}))}}{2 e^{k \alpha^{\prime} \varphi((\underline{1}))}} \asymp e^{\alpha k} .
$$

The next observation explains why we can restrict the discussion to the above two cases in (2.1). For this we define

$$
\begin{aligned}
& \mathcal{D}_{\sharp}:=\pi_{S}\left(\left\{x \in \Sigma: \lim _{n \rightarrow \infty} \mathcal{S}_{n} \chi(x)=-\infty\right\}\right), \\
& \mathcal{D}_{\sharp}^{*}=\mathcal{D}_{\sharp} \backslash \pi_{S}\left(\left\{\left(x_{1} x_{2} \ldots\right) \in \Sigma: \exists n \in \mathbb{N} \exists i \in\{1, d\} \forall k \geq n: x_{k}=i\right\}\right) .
\end{aligned}
$$

LEMma 2.7. If we are in neither of the two cases in (2.1), then

$$
\mathcal{D}_{\sim} \cap \mathcal{D}_{\sharp}^{*}=\emptyset .
$$

Proof. Let $x=\left(x_{1} x_{2} \ldots\right) \in \Sigma$ be such that $\xi:=\pi_{S}(x) \in \mathcal{D}_{\sharp}^{*}$. Assume that $x$ has a strict $j$-block of length $k$ at the $n$th level, $j \in\{1, d\}$. We have to distinguish two cases. The first is

$$
\frac{\psi((\underline{1}))}{\varphi((\underline{1}))} \geq 1 \quad \text { and } \quad \frac{\psi((\underline{d}))}{\varphi((\underline{d}))} \geq 1 .
$$

Then $e^{\psi((\underline{i}))} \leq e^{\varphi((\underline{i}))}$ for $i \in\{1, d\}$, and clearly $\frac{e^{l \psi((\underline{1}))}+e^{k \psi((\underline{d}))}}{e^{l \varphi((\underline{1}))}+e^{k \varphi(\underline{(\underline{l}))}} \leq 1 \text { for all }}$ $k, l \in \mathbb{N}$. By combining this observation with Proposition 2.1 and Lemma 2.2, it follows that $\xi \in \mathcal{D}_{0}$, and hence $\xi \notin \mathcal{D}_{\sim}$. 
The second case is

$$
\frac{\psi((\underline{1}))}{\varphi((\underline{1}))}=\frac{\psi((\underline{d}))}{\varphi((\underline{d}))}<1 .
$$

As in the proof of Lemma 2.6, it then follows that for each $i \in\{1, d\} \backslash\{j\}$ and for all $l, k \in \mathbb{N}$, we have

$$
\frac{e^{l \psi((\underline{i}))}+e^{k \psi((\underline{j}))}}{e^{l \varphi((\underline{i}))}+e^{k \varphi((\underline{j}))}} \leq 2 e^{k \chi((\underline{j}))} .
$$

Therefore, for each $\eta \in \pi_{S}\left(\left[x_{1} \ldots x_{n-1}\right] \backslash\left[x_{1} \ldots x_{n}\right]\right)$ we have

$$
D_{\Theta}(\xi, \eta) \ll e^{\mathcal{S}_{n+k} \chi(x)} .
$$

Using this observation and Lemma 2.2, we find that the derivative of $\Theta$ at $\xi$ is equal to 0 , and hence $\xi \notin \mathcal{D}$.

Proposition 2.8. Assume that we are in Case 1 of (2.1) and let $x=$ $\left(x_{1} x_{2} \ldots\right) \in \Sigma$ be such that $\xi:=\pi_{S}(x) \in \mathcal{D}_{\sharp}^{*}$. Then $\xi \in \mathcal{D}_{\sim}$ if and only if there exist strictly increasing sequences $\left(n_{m}\right)_{m \in \mathbb{N}}$ and $\left(k_{m}\right)_{m \in \mathbb{N}}$ of positive integers such that $x$ has a strict $d$-block of length $k_{m}$ at the $n_{m}$ th level for each $m \in \mathbb{N}$, and

$$
e^{\mathcal{S}_{n_{m}} \chi(x)+k_{m} \alpha} \gg 1 \quad \text { for all } m \in \mathbb{N} .
$$

Proof. By Lemma 2.4 there exists a sequence $\left(\eta_{n}\right)_{n \in \mathbb{N}}$ such that

$$
\lim _{n \rightarrow \infty} D_{\Theta}\left(\xi, \eta_{n}\right)=0 \text {. }
$$

Now, for the "if" part, assume that $\xi$ has strict $d$-blocks as specified in the proposition. For each $m \in \mathbb{N}$, choose $\eta_{m}^{\prime}$ in the interval $\pi_{S}\left(\left[x_{1} \ldots\right.\right.$ $\left.\left.x_{n_{m}}\left(x_{n_{m}+1}+1\right) \underline{1}_{l_{m}} a\right]\right)$, where $a \in A \backslash\{1\}$ and $l_{m}:=k_{m} \alpha / \chi((\underline{1}))$. Combining Proposition 2.1, the second part of Lemma 2.6, and the fact that $\exp \left(\mathcal{S}_{n_{m}} \chi(x)+k_{m} \alpha\right) \gg 1$, we then obtain, for all $m \in \mathbb{N}$,

$$
D_{\Theta}\left(\xi, \eta_{m}^{\prime}\right) \asymp e^{\mathcal{S}_{n_{m}} \chi(x)} \frac{e^{k_{m} \psi((\underline{d}))}+e^{l_{m} \psi((\underline{1}))}}{e^{k_{m} \varphi((\underline{d}))}+e^{l_{m} \varphi((\underline{1}))}} \asymp e^{\mathcal{S}_{n_{m}} \chi(x)+k_{m} \alpha} \gg 1 .
$$

Combining this with the observation at the beginning of the proof, it follows that $\xi \in \mathcal{D}_{\sim}$.

For the "only if" part, let $x=\left(x_{1} x_{2} \ldots\right) \in \Sigma$ with $\xi:=\pi_{S}(x) \in \mathcal{D}_{\sim} \cap D_{\sharp}^{*}$. Then there exists a sequence $\left(\eta_{m}\right)_{m \in \mathbb{N}}$ in $\mathcal{U}$ and a strictly increasing sequence $\left(n_{m}\right)_{m \in \mathbb{N}}$ in $\mathbb{N}$ such that $\eta_{m} \in \pi_{S}\left(\left[x_{1} \ldots x_{n_{m}}\right]\right)$ for all $m \in \mathbb{N}$ and

$$
\liminf _{m \rightarrow \infty} D_{\Theta}\left(\xi, \eta_{m}\right)>0 \text {. }
$$

Using Proposition 2.1 and Lemma 2.6, it follows that if $x$ has a $d$-block of length $k_{m}$ at the $n_{m}$ th level, then for all $l, m \in \mathbb{N}$,

$$
D_{\Theta}\left(\xi, \eta_{m}\right) \ll e^{\mathcal{S}_{n_{m}} \chi(x)} \frac{e^{l \psi((\underline{1}))}+e^{k_{m} \psi((\underline{d}))}}{e^{l \varphi((\underline{1}))}+e^{k_{m} \varphi((\underline{d}))}} \ll e^{\mathcal{S}_{n_{m}} \chi(x)+\alpha k_{m}} .
$$


Since $\liminf \operatorname{in}_{m \rightarrow \infty} D_{\Theta}\left(\xi, \eta_{m}\right)>0$, it follows that

$$
\liminf _{m \rightarrow \infty} e^{\mathcal{S}_{n_{m}} \chi(x)+\alpha k_{m}}>0,
$$

and therefore $e^{\mathcal{S}_{n_{m}} \chi(x)+\alpha k_{m}} \gg 1$ for all $m \in \mathbb{N}$.

2.2. The upper bound. We start by observing that

$$
\limsup _{n \rightarrow \infty} e^{\mathcal{S}_{n} \chi}>0 \Rightarrow \limsup _{n \rightarrow \infty} \frac{\mathcal{S}_{n} \varphi}{\mathcal{S}_{n} \psi} \geq 1 \text {. }
$$

This implies that

$$
\operatorname{dim}_{\mathrm{H}}\left(\left\{\limsup _{n \rightarrow \infty} e^{\mathcal{S}_{n} \chi}>0\right\}\right) \leq \operatorname{dim}_{\mathrm{H}}\left(\left\{\limsup _{n \rightarrow \infty} \frac{\mathcal{S}_{n} \varphi}{\mathcal{S}_{n} \psi} \geq 1\right\}\right)=\widehat{\beta}(1) .
$$

Here, the final equality holds since the Lyapunov dimension spectrum $s \mapsto$ $\widehat{\beta}(s) / s$ is decreasing in a neighbourhood of 1 . Since the sets $\mathcal{D}_{\infty}$ and $\mathcal{D}_{\sim} \cap$ $\left\{\limsup _{n \rightarrow \infty} e^{\mathcal{S}_{n} \chi}>0\right\}$ are contained in $\left\{\limsup _{n \rightarrow \infty} e^{\mathcal{S}_{n} \chi}>0\right\}$, the observation above gives the upper bound $\widehat{\beta}(1)$ for the Hausdorff dimension of each of these two sets.

Since $\lim _{n \rightarrow \infty} \exp \left(\mathcal{S}_{n} \chi(x)\right)=0$ implies $\pi_{S}(x) \in \mathcal{D}_{\sharp}^{*}$, except for the countable set of end points of all refinements of the Markov partition, it is sufficient to show that

$$
\operatorname{dim}_{H}\left(\mathcal{D}_{\sim} \cap \mathcal{D}_{\sharp}^{*}\right) \leq \widehat{\beta}(1) .
$$

This part of the proof is inspired by the arguments given in [11]. First note that it is sufficient to show that

$$
\operatorname{dim}_{\mathrm{H}}\left(\mathcal{D}_{\sim} \cap \mathcal{D}_{\sharp}^{*}\right) \leq \widetilde{\beta}(s) \text { for all } s \leq 1 .
$$

In a nutshell, the idea is to show that for each $s \leq 1$ there is a suitable covering of $\mathcal{D}_{\sim} \cap \mathcal{D}_{\sharp}^{*}$ which will then be used to deduce that the $\widetilde{\beta}(s)$-dimensional Hausdorff measure of $\mathcal{D}_{\sim} \cap \mathcal{D}_{\sharp}^{*}$ is finite.

If we are in neither of the two cases of $(2.1)$, we have $\mathcal{D}_{\sim} \cap \mathcal{D}_{\sharp}^{*}=\emptyset$ by Lemma 2.7. Hence for ease of exposition, throughout the remaining part of this section we will again assume that we are in Case 1 of (2.1). Clearly, the considerations for Case 2 are completely analogous, and will therefore be omitted. Let us first introduce the stopping time $\tau_{t}$ with respect to $\chi$ on $\pi_{S}^{-1}\left(\mathcal{D}_{\sharp}^{*}\right)$ by

$$
\tau_{t}(x):=\inf \left\{k \in \mathbb{N}: \mathcal{S}_{k} \chi(x)<-t\right\} \quad \text { for all } t>0, x \in \pi_{S}^{-1}\left(\mathcal{D}_{\sharp}^{*}\right) .
$$

For each $n \in \mathbb{N}$ fix a partition $\mathcal{C}_{n}$ of $\pi_{S}^{-1}\left(\mathcal{D}_{\sharp}^{*}\right)$ consisting of cylinder sets $[\omega]$ with the following property:

$$
\left|\mathcal{S}_{\tau_{n}(x)} \chi(x)+n\right| \ll 1 \quad \text { for all } x \in[\omega] .
$$

Moreover, for $\epsilon>0$ we define

$$
\mathcal{C}_{n}(\epsilon):=\left\{\left[\omega \underline{d}_{n_{\epsilon}}\right]:[\omega] \in \mathcal{C}_{n}\right\},
$$


where $n_{\epsilon}$ is given by $n_{\epsilon}:=\lfloor n(1-\epsilon) / \alpha\rfloor$. For $s \in(0,1)$ we choose $\epsilon>0$ such that

$$
(1-\epsilon) \widetilde{\beta}(s)>(-\chi((\underline{1})) / \varphi((\underline{1}))) \beta(s) .
$$

This is possible, since on the one hand we have $\widetilde{\beta}(s)-\beta(s)=s>0$ and hence $\widetilde{\beta}(s)>\beta(s)$ for all $s \in(0,1)$. On the other hand, the fact that $\psi<0$ immediately implies that $-\chi((\underline{1})) / \varphi((\underline{1}))<1$. Recall that we are assuming that Case 1 of $(2.1)$ holds, and therefore $-\chi((\underline{1})) / \varphi((\underline{1}))=$ $1-\psi((\underline{1})) / \varphi((\underline{1}))>0$. It then follows that

$$
\begin{aligned}
\sum_{n \in \mathbb{N}} \sum_{C \in \mathcal{C}_{n}(\epsilon)}(\operatorname{diam}(C))^{\widetilde{\beta}(s)} \asymp \sum_{n \in \mathbb{N}} \sum_{C \in \mathcal{C}_{n}(\epsilon)} e^{\sup _{x \in C} \widetilde{\beta}(s) \mathcal{S}_{\tau_{n}(x)+n_{\epsilon}} \varphi(x)} \\
\ll \sum_{n \in \mathbb{N}} e^{n(1-\epsilon) \widetilde{\beta}(s) \varphi((\underline{d})) / \alpha} \sum_{C \in \mathcal{C}_{n}} e^{\widetilde{\beta}(s) \sup _{x \in C} \mathcal{S}_{\tau_{n}(x)} \varphi(x)} \\
\asymp \sum_{n \in \mathbb{N}} e^{n(1-\epsilon) \widetilde{\beta}(s) \varphi((\underline{d})) / \alpha+n \beta(s)} \sum_{C \in \mathcal{C}_{n}} e^{\sup _{x \in C} \mathcal{S}_{\tau_{n}(x)}(\widetilde{\beta}(s) \varphi(x)+\beta(s) \chi(x))} \\
\ll \sum_{n \in \mathbb{N}}\left(e^{(1-\epsilon) \widetilde{\beta}(s) \varphi((\underline{d})) / \alpha+\beta(s)}\right)^{n}<\infty .
\end{aligned}
$$

Here we have used the Gibbs property

$$
\sum_{C \in \mathcal{C}_{n}} e^{\sup _{x \in C} \mathcal{S}_{\tau_{n}(x)}(\widetilde{\beta}(s) \varphi(x)+\beta(s) \chi(x))}=\sum_{C \in \mathcal{C}_{n}} e^{\sup _{x \in C} \mathcal{S}_{\tau_{n}(x)}(s \varphi(x)+\beta(s) \psi(x))} \ll 1
$$

of the Gibbs measure $\mu_{s}$ and the fact that

$$
(1-\epsilon) \widetilde{\beta}(s) \varphi((\underline{d})) / \alpha+\beta(s)=(1-\epsilon) \widetilde{\beta}(s) \varphi((\underline{1})) / \chi((\underline{1}))+\beta(s)<0 .
$$

Thus, for the limsup-set

$$
C_{\infty}(\epsilon):=\left\{\xi \in \mathcal{U}: \xi \in \pi_{S}\left(\mathcal{C}_{n}(\epsilon)\right) \text { for infinitely many } n \in \mathbb{N}\right\}
$$

we now have

$$
\operatorname{dim}_{\mathrm{H}}\left(C_{\infty}(\epsilon)\right) \leq \min _{s \in(0,1)} \widetilde{\beta}(s)=\widehat{\beta}(1) .
$$

Hence, it remains to show that

$$
\mathcal{D} \sim \cap \mathcal{D}_{\sharp}^{*} \subset C_{\infty}(\epsilon) \quad \text { for all } \epsilon>0 .
$$

For this, let $x \in \Sigma$ be such that $\xi:=\pi_{S}(x) \in \mathcal{D}_{\sim} \cap \mathcal{D}_{\sharp}^{*}$. By Proposition 2.8, there exist strictly increasing sequences $\left(n_{m}\right)_{m \in \mathbb{N}}$ and $\left(k_{m}\right)_{m \in \mathbb{N}}$ of positive integers such that $x$ has a $d$-block of length $k_{m}$ at the $n_{m}$ th level and

$$
e^{\mathcal{S}_{n_{m}} \chi(x)+k_{m} \alpha} \gg 1 \quad \text { for each } m \in \mathbb{N} \text {. }
$$

By setting $\ell\left(n_{m}\right):=\left\lfloor\mathcal{S}_{n_{m}} \chi(x)\right\rfloor$, it follows $\exp \left(k_{m}\right) \gg \exp \left(-\ell\left(n_{m}\right) / \alpha\right)$. Hence, for each $\epsilon>0$ and for each $m$ sufficiently large, we have $k_{m} \geq$ $-\ell\left(n_{m}\right)(1-\epsilon) / \alpha$. It follows that $\xi \in C_{\infty}(\epsilon)$, which finishes the proof of the upper bound. 
2.3. The lower bound. In this section we show that the Hausdorff dimension of each of the sets $\mathcal{D}_{\sim}$ and $\mathcal{D}_{\infty}$ is bounded below by $\widetilde{\beta}\left(s_{0}\right)$. Clearly, combining this with the results of the previous section will then complete the proof of Theorem 1.1. Let us begin by showing that

$$
\operatorname{dim}_{\mathrm{H}}\left(\mathcal{D}_{\sim}\right) \geq \widetilde{\beta}\left(s_{0}\right) .
$$

Recall that $\mu_{s}$ refers to the equilibrium measure for the potential $s \varphi+\beta(s) \psi$, and that $s_{0}$ is chosen so that

$$
\beta^{\prime}\left(s_{0}\right)=-\frac{\int \varphi d \mu_{s_{0}}}{\int \psi d \mu_{s_{0}}}=-1 .
$$

This implies that

$$
0=\int \psi d \mu_{s_{0}}-\int \varphi d \mu_{s_{0}}=\int \chi d \mu_{s_{0}} .
$$

By the variational principle, we have

$$
h\left(\mu_{s_{0}}\right)+s_{0} \int \varphi d \mu_{s_{0}}+\beta\left(s_{0}\right) \int \psi d \mu_{s_{0}}=0,
$$

and hence

$$
\frac{h\left(\mu_{s_{0}}\right)}{-\int \varphi d \mu_{s_{0}}}=\beta\left(s_{0}\right)+s_{0}=\widetilde{\beta}\left(s_{0}\right) .
$$

Since we are in the expanding case, we can use Young's formula (see [14, 23]) to deduce that $\operatorname{dim}_{\mathrm{H}}\left(\pi_{S}\left(\mu_{s_{0}}\right)\right)=\widetilde{\beta}\left(s_{0}\right)$. The lower bound for the Hausdorff dimension of $\mathcal{D}_{\sim}$ now follows from combining Corollary 2.5 with the following lemma.

Lemma 2.9. For $\mu_{s_{0}}$-almost every $x \in \Sigma$ we have

$$
\liminf _{n \rightarrow \infty} e^{\mathcal{S}_{n} \chi(x)}=0 \quad \text { and } \quad \limsup _{n \rightarrow \infty} e^{\mathcal{S}_{n} \chi(x)}=\infty .
$$

Proof. Note that $\int \chi d \mu_{s_{0}}=0$. Thus, by the law of the iterated logarithm [6] there exists a constant $C>0$ such that for $\mu_{s_{0}}$-almost all $x \in \Sigma$ we have

$$
\liminf _{n \rightarrow \infty} \frac{\mathcal{S}_{n} \chi(x)}{\sqrt{n \log \log n}}=-C \text { and } \limsup _{n \rightarrow \infty} \frac{\mathcal{S}_{n} \chi(x)}{\sqrt{n \log \log n}}=C .
$$

From this the conclusion follows.

Lemma 2.9 implies that $\pi_{S}(x) \in \mathcal{D}_{\sim}$ for $\mu_{s_{0}}$-almost every $x \in \Sigma$, and hence

$$
\operatorname{dim}_{\mathrm{H}}\left(\mathcal{D}_{\sim}\right) \geq \operatorname{dim}_{\mathrm{H}}\left(\pi_{S}\left(\mu_{s_{0}}\right)\right)=\widetilde{\beta}\left(s_{0}\right) .
$$

Therefore, it remains to show that

$$
\operatorname{dim}_{H}\left(\mathcal{D}_{\infty}\right) \geq \widetilde{\beta}\left(s_{0}\right) .
$$

For this, we consider the set of the equilibrium measures $\left\{\mu_{s}: s>s_{0}\right\}$.

Lemma 2.10. For $s>s_{0}$, we have

$$
\int \chi d \mu_{s}>0
$$


Proof. Since $\beta$ is strictly convex, $s>s_{0}$ implies that $\beta^{\prime}(s)>-1$. This gives

and hence $\int \chi d \mu_{s}>0$.

$$
\frac{\int \varphi d \mu_{s}}{\int \psi d \mu_{s}}=-\beta^{\prime}(s)<1
$$

Lemma 2.10 implies that for $\mu_{s}$-almost every $x \in \Sigma$ we have (recall that we are assuming that $s>s_{0}$ )

$$
\lim _{n \rightarrow \infty} e^{\mathcal{S}_{n} \chi(x)}=\infty .
$$

For the following lemma we introduce the following notations. For $x=$ $\left(x_{1} x_{2} \ldots\right) \in \Sigma, k, n \in \mathbb{N}$ and $i \in\{1, d\}$, let $k_{n}(x):=k$ if $x$ has an $i$ block of length $k$ at the $n$th level, and set $k_{n}(x):=0$ if $x_{n+1} \notin\{1, d\}$. We then have the following routine Khinchin-type estimate, where $\kappa_{i, s}:=$ $-(s \varphi((\underline{i}))+\beta(s) \psi((\underline{i})))^{-1}>0$ and $\kappa_{s}:=\min \left\{\kappa_{i, s}: i=1, d\right\}$.

Lemma 2.11. For $\mu_{s}$-almost every $x \in \Sigma$, we have

$$
\limsup _{n \rightarrow \infty} \frac{k_{n}(x)}{\log n} \leq \kappa_{s} .
$$

Proof. Let $\mathcal{C}_{n}^{*}:=\left\{[\omega]: \omega \in A^{n}\right\}$ and recall

$$
\sum_{C \in \mathcal{C}_{n}^{*}} \exp \left(\sup _{x \in C} \mathcal{S}_{n}(s \varphi+\beta(s) \psi)(x)\right) \asymp 1
$$

for all $n \in \mathbb{N}$. For $\epsilon>0$, let $k_{\epsilon, i, n}:=\left\lfloor(1+\epsilon) \kappa_{i, s} \log n\right\rfloor$. Then

$$
\sum_{n \in \mathbb{N}} \sum_{\left[x_{1} \ldots x_{n}\right] \in \mathcal{C}_{n}^{*}} e^{\sup _{x \in\left[x_{1} \ldots x_{n} \underline{i}_{\epsilon, i, n}\right]} \mathcal{S}_{n+k_{\epsilon, i, n}}(s \varphi+\beta(s) \psi)(x)} \ll \sum_{n \in \mathbb{N}} n^{-(1+\epsilon)} .
$$

Hence, by the Borel-Cantelli Lemma, the set of elements in $\Sigma$ which lie in cylinder sets of the form $\left[x_{1} \ldots x_{n} \underline{i}_{k_{\epsilon, i, n}}\right]$ for infinitely many $n \in \mathbb{N}$ has $\mu_{s}$-measure equal to zero. By passing to the complement of this limsup-set, the statement in the lemma follows.

We can now complete the proof of Theorem 1.1 as follows. By Lemma 2.3, there exists a constant $c>0$ such that for each $x=\left(x_{1} x_{2} \ldots\right) \in \Sigma$ and for each sequence $\left(\eta_{n}\right)_{n}$ in $\mathcal{U}$ tending to $\xi:=\pi_{S}(x)$,

$$
\liminf _{n \rightarrow \infty} D_{\Theta}\left(\xi, \eta_{n}\right) \geq c \liminf _{n \rightarrow \infty} e^{\mathcal{S}_{n} \chi(x)} e^{-k_{n} \delta} .
$$

Moreover, using Lemma 2.10 and the ergodicity of $\mu_{s}$, it follows that for $\mu_{s}$-almost every $x \in \Sigma$ we have

$$
\lim _{n \rightarrow \infty} \frac{1}{n} \mathcal{S}_{n} \chi(x)=\int \chi d \mu_{s}=: c_{\chi}(x)>0 .
$$

Combining this with Lemma 2.11, it follows that for $\mu_{s}$-almost every $x \in \Sigma$, with $\xi=\pi_{S}(x)$, we have

$$
\liminf _{n \rightarrow \infty} D_{\Theta}\left(\xi, \eta_{n}\right) \geq c \liminf _{n \rightarrow \infty} e^{\mathcal{S}_{n} \chi(x)} e^{-\delta k_{n}(x)} \geq c \liminf _{n \rightarrow \infty} e^{n c_{\chi}(x)} n^{-\delta \kappa_{s}}=\infty .
$$


This implies

$$
\lim _{n \rightarrow \infty} D_{\Theta}\left(\xi, \eta_{n}\right)=\infty \quad \mu_{s} \text {-almost everywhere. }
$$

Since $\pi_{S}$ is bijective except on a countable number of points, we now conclude that for all $s>s_{0}$ we have

$$
\operatorname{dim}_{\mathrm{H}}\left(\mathcal{D}_{\infty}\right) \geq \operatorname{dim}_{\mathrm{H}}\left(\pi_{S}\left(\mu_{s}\right)\right)=-\widehat{\beta}\left(-\beta^{\prime}(s)\right) / \beta^{\prime}(s) .
$$

To complete the proof, simply note that $-\widehat{\beta}\left(-\beta^{\prime}(s)\right) / \beta^{\prime}(s) \nearrow \widetilde{\beta}\left(s_{0}\right)$ for $s \searrow s_{0}$. This finishes the proof of Theorem 1.1.

3. Proof of Theorem 1.2. If $(\mathcal{U}, S)$ and $(\mathcal{U}, T)$ are $C^{1+\epsilon}$ conjugate, then clearly $\mathcal{D}_{\sim}(S, T)=\emptyset$, and hence $\operatorname{dim}_{\mathrm{H}}\left(\mathcal{D}_{\sim}(S, T)\right)=0$. This gives one direction of the equivalence in Theorem 1.2.

For the other direction, assume that $\operatorname{dim}_{H}\left(\mathcal{D}_{\sim}(S, T)\right)=0$. Then Theorem 1.1 implies that $\varphi$ and $\psi$ are cohomologically dependent. That is, there exist $b, c \in \mathbb{R} \backslash\{0\}$ and a Hölder continuous function $u: \Sigma \rightarrow \mathbb{R}$ such that

$$
b \varphi+c \psi=u-u \circ \sigma .
$$

Then for all $s \in \mathbb{R}$,

$$
P(s \varphi-(b / c) \beta(s) \varphi)=P((s-(b / c) \beta(s)) \varphi)=0,
$$

and hence $\beta(s)=(s-1) c / b$. Combining this with $\beta(0)=1$ shows that $b / c=-1$, and therefore

$$
\psi-\varphi=\chi=v-v \circ \sigma
$$

for some Hölder continuous function $v: \Sigma \rightarrow \mathbb{R}$. Note that in particular $\psi((\underline{i}))=\varphi((\underline{i}))$ for each $i \in\{1, d\}$. Combining this with Proposition 2.1, it follows that uniformly for all $\xi, \eta \in \mathcal{U}$ we have

$$
D_{\Theta}(\xi, \eta) \asymp 1 \text {. }
$$

This shows that there exists a constant $c_{0}>1$ such that for all $\xi \in \mathcal{U}$,

$$
c_{0}^{-1}<\liminf _{\eta \rightarrow \xi} D_{\Theta}(\xi, \eta) \leq \limsup _{\eta \rightarrow \xi} D_{\Theta}(\xi, \eta)<c_{0} .
$$

Since the derivative of $\Theta$ exists Lebesgue almost everywhere, it follows that $\Theta^{\prime}(\xi)$ is uniformly bounded away from zero and infinity for Lebesgue almost every $\xi \in \mathcal{U}$. We can now complete the proof by an argument similar to the one in [3] (see the introduction for a statement of the main result of [3]). We have split the discussion into four steps. Here, for $c \in \mathbb{R}$, we let $f_{c}: \mathbb{R} \rightarrow \mathbb{R}$ denote the multiplication map given by $x \mapsto c x$, and we set $\sigma_{0}:=S^{\prime}(0)=T^{\prime}(0)$. Note that, since $\psi((\underline{1}))=\varphi((\underline{1}))$, we clearly have $S^{\prime}(0)=T^{\prime}(0)$.

Linearisation. For each $n \in \mathbb{N}$, let $S_{1}^{-n}$ and $T_{1}^{-n}$ denote the inverse branches of $S^{n}$ and $T^{n}$, respectively, such that 0 is contained in $S_{1}^{-n}(\mathcal{U})$ and 
$T_{1}^{-n}(\mathcal{U})$. Using the bounded distortion property and the fact that $\left(S_{1}^{-n}\right)^{\prime}$ and $\left(T_{1}^{-n}\right)^{\prime}$ are uniformly Hölder continuous, we see, by Arzelà-Ascoli, that there exist subsequences of $\left(f_{\sigma_{0}^{n} \circ S_{1}^{-n}}\right)_{n \in \mathbb{N}}$ and $\left(f_{\sigma_{0}^{n} \circ T_{1}^{-n}}\right)_{n \in \mathbb{N}}$ which converge uniformly on $\mathcal{U}$ to $C^{1+\epsilon}$ diffeomorphisms $\gamma_{S}$ and $\gamma_{T}$ respectively. Note that clearly $\gamma_{S} \circ S=f_{\sigma_{0}} \circ \gamma_{S}$ and $\gamma_{T} \circ T=f_{\sigma_{0}} \circ \gamma_{T}$.

Differentiation. The uniform Hölder continuity of $\left(S_{1}^{-n}\right)^{\prime}$ and $\left(T_{1}^{-n}\right)^{\prime}$ and the fact that the conjugacy $\Theta$ is bi-Lipschitz imply that the right derivative of $\Theta$ at zero exists and that it has a finite and positive value.

Localisation. We have

$$
\begin{aligned}
f_{\sigma_{0}} \circ \gamma_{T} \circ \Theta \circ \gamma_{S}^{-1} & =\gamma_{T} \circ T \circ \Theta \circ \gamma_{S}^{-1}=\gamma_{T} \circ \Theta \circ S \circ \gamma_{S}^{-1} \\
& =\gamma_{T} \circ \Theta \circ \gamma_{S}^{-1} \circ \gamma_{S} \circ S \circ \gamma_{S}^{-1} \\
& =\gamma_{T} \circ \Theta \circ \gamma_{S}^{-1} \circ f_{\sigma_{0}} \circ \gamma_{S} \circ \gamma_{S}^{-1} \\
& =\gamma_{T} \circ \Theta \circ \gamma_{S}^{-1} \circ f_{\sigma_{0}},
\end{aligned}
$$

which shows that $\gamma_{T} \circ \Theta \circ \gamma_{S}^{-1}$ commutes with $f_{\sigma_{0}}$. Using this and the differentiability of $\Theta$ at 0 , we deduce that $\gamma_{T} \circ \Theta \circ \gamma_{S}^{-1}=\left(f_{\sigma_{0}}\right)^{n} \circ \gamma_{T} \circ \Theta \circ \gamma_{S}^{-1} \circ\left(f_{1 / \sigma_{0}}\right)^{n}$ on the domain of $\gamma_{S}$. Therefore, for $\xi$ in this domain,

$$
\gamma_{T} \circ \Theta \circ \gamma_{S}^{-1}(\xi)=\frac{\gamma_{T} \circ \Theta \circ \gamma_{S}^{-1}\left(\sigma_{0}^{-n} \cdot \xi\right)}{\sigma_{0}^{-n} \cdot \xi} \cdot \xi \rightarrow \kappa_{0} \cdot \xi \quad \text { as } n \rightarrow \infty,
$$

where $\kappa_{0}>0$ denotes the right derivative of $\gamma_{T} \circ \Theta \circ \gamma_{S}^{-1}$ at zero. It now follows that there exists $\delta>0$ such that $\left.\Theta\right|_{[0, \delta]}$ is a $C^{1+\epsilon}$ diffeomorphism.

Globalisation. Let $n \in \mathbb{N}$ be chosen such that $S_{1}^{-n}(\mathcal{U}) \subset[0, \delta]$. Since $\Theta=T^{n} \circ \Theta \circ S_{1}^{-n}$, it follows that $\Theta: \mathcal{U} \rightarrow \mathcal{U}$ is a $C^{1+\epsilon}$ diffeomorphism. This completes the proof of the main part of Theorem 1.2.

In order to prove the Hölder regularity of $\Theta$, as claimed in part (2) of Theorem 1.2, let $\xi, \eta \in \mathcal{U}$ and put $\rho:=\sup _{s \in \mathbb{R}}\left(-\beta^{\prime}(s)\right)>0$. Clearly, $\mathcal{S}_{n} \psi(x) / \mathcal{S}_{n} \varphi(x)>1 / \rho$ for all $x \in \Sigma$ and $n \in \mathbb{N}$. Without loss of generality, we can assume that $\xi=\pi_{S}\left(x_{1} x_{2} \ldots\right)<\eta=\pi_{S}\left(y_{1} y_{2} \ldots\right)$ and $\eta \in$ $\pi_{S}\left(\left[x_{1} \ldots x_{n}\right] \backslash\left[x_{1} \ldots x_{n+1}\right]\right)$. Moreover, let us only consider the case where $x$ has a strict $d$-block of length $k$ at the $n$th level and $y$ has a strict 1-block of length $l$ at the $(n+1)$ th level, for some $k, l, n \in \mathbb{N}$. Then there exists a uniform constant $C>0$ such that

$$
\begin{aligned}
|\Theta(\xi)-\Theta(\eta)| & \leq C\left(e^{\mathcal{S}_{n+k} \psi(x)}+e^{\mathcal{S}_{n+l} \psi(y)}\right) \\
& =C\left(e^{\frac{\mathcal{S}_{n+k} \psi(x)}{\mathcal{S}_{n+k} \varphi(x)} \mathcal{S}_{n+k} \varphi(x)}+e^{\frac{\mathcal{S}_{n+l} \psi(y)}{\mathcal{S}_{n+l} \varphi(y)} \mathcal{S}_{n+l} \varphi(y)}\right) \\
& \leq C\left(e^{\rho^{-1} \mathcal{S}_{n+k} \varphi(x)}+e^{\rho^{-1} \mathcal{S}_{n+l} \varphi(y)}\right) \\
& \leq 2 C\left(e^{\mathcal{S}_{n+k} \varphi(x)}+e^{\mathcal{S}_{n+l} \varphi(y)}\right)^{1 / \rho} \leq 2 C|\xi-\eta|^{1 / \rho} .
\end{aligned}
$$


Note that if one of the blocks has infinite word length, then one has to use approximations of this block by words of finite lengths.

It remains to show that if $S$ and $T$ are cohomologically independent, then $\Theta$ has to be singular with respect to the Lebesgue measure $\lambda$. For this, note that on the unit interval without the boundary points of all refinements of the Markov partition we have $\Theta=\pi_{T} \circ \pi_{S}^{-1}$. Therefore, it is sufficient to show that the measure $\lambda \circ \Theta$, whose distribution function is equal to $\Theta$, is singular with respect to $\lambda$. Since $\mu_{\psi} \circ \pi_{T}^{-1}, \mu_{\varphi} \circ \pi_{S}^{-1}$ and $\lambda$ are all in the same measure class, it follows that $\lambda \circ \Theta$ is absolutely continuous with respect to $\mu_{\psi} \circ \pi_{T}^{-1} \circ \pi_{T} \circ \pi_{S}^{-1}=\mu_{\psi} \circ \pi_{S}^{-1}$. On the other hand, since $S$ and $T$ are cohomologically independent, $\mu_{\psi} \circ \pi_{S}^{-1}$ is singular with respect to $\mu_{\varphi} \circ \pi_{S}^{-1}$. This finishes the proof of Theorem 1.2.

4. Examples. In this section we consider two families of examples: the Salem family and the sine family. For the Salem family we will see in Section 5 that it gives rise to conjugacies whose sets of nondifferentiability have Hausdorff dimensions arbitrarily close to zero.

ExAmple 1 (the Salem family). Let us consider a class of examples studied by Salem in [20]. Namely, we consider the family of conjugacy maps $\left\{\Theta_{\tau}: \tau \in(0,1) \backslash\{1 / 2\}\right\}$ which arises from the following endomorphisms of $\mathcal{U}$. For $\xi \in \mathcal{U}$, we define

$$
T(\xi):=2 \xi \bmod 1 \quad \text { and } \quad S_{\tau}(\xi):= \begin{cases}\xi / \tau & \text { if } 0 \leq \xi \leq \tau, \\ (\xi-\tau) /(1-\tau) & \text { if } \tau<\xi \leq 1 .\end{cases}
$$

The maps $\Theta_{\tau}:[0,1] \rightarrow[0,1]$ are then given by $T \circ \Theta_{\tau}=\Theta_{\tau} \circ S_{\tau}$. One

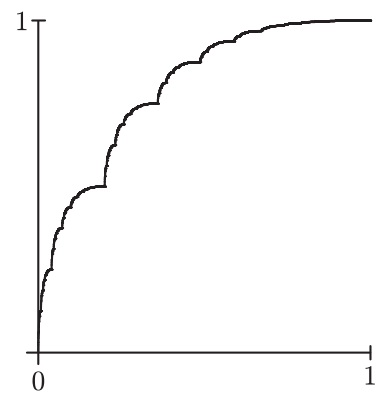

(a) $\tau=1 / 5$

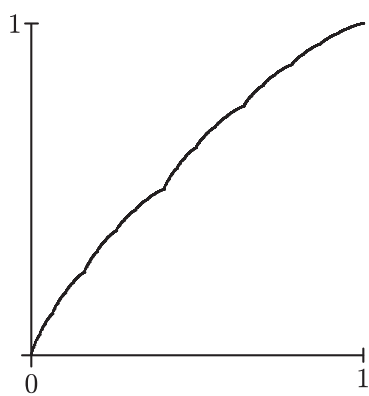

(b) $\tau=2 / 5$

Fig. 1. The conjugating map $\Theta_{\tau}$ for the Salem case

immediately verifies that $\Theta_{\tau}$ is strictly monotone and has the property that $\Theta_{\tau}^{\prime}(\xi)=0$ for Lebesgue almost every $\xi \in \mathcal{U}$. Note that the conjugacies considered in [20] are in fact dual to the ones which we consider here. However, this has no effect on the Hausdorff dimension of $\mathcal{D}_{\sim}\left(S_{\tau}, T\right)$ (see Re- 
mark 1.6(2)), and our conjugacies have the advantage that they allow us to determine $\beta_{\tau}$ and $\operatorname{dim}_{\mathrm{H}}\left(\mathcal{D}_{\sim}\left(S_{\tau}, T\right)\right)$ rather explicitly. For this, first note that in the current situation the potential functions $\varphi_{\tau}$ and $\psi$ are given for $x=\left(x_{1} x_{2} \ldots\right) \in \Sigma$ by

$$
\psi(x)=-\log 2 \quad \text { and } \quad \varphi_{\tau}(x)= \begin{cases}\log \tau & \text { if } x_{1}=1 \\ \log (1-\tau) & \text { if } x_{1}=2\end{cases}
$$

The function $\beta_{\tau}$ is defined implicitly by $P\left(s \varphi_{\tau}+\beta(t) \psi\right)=0$. Since we have $\exp \left(s \log \tau-\beta_{\tau}(s) \log 2\right)+\exp \left(s \log (1-\tau)-\beta_{\tau}(s) \log 2\right)=1$, an elementary calculation gives that $\beta_{\tau}$ is given explicitly by

$$
\beta_{\tau}(s)=\frac{P\left(s \varphi_{\tau}\right)}{\log 2}=\log _{2}\left(\tau^{s}+(1-\tau)^{s}\right) \quad \text { for each } s \in \mathbb{R} .
$$

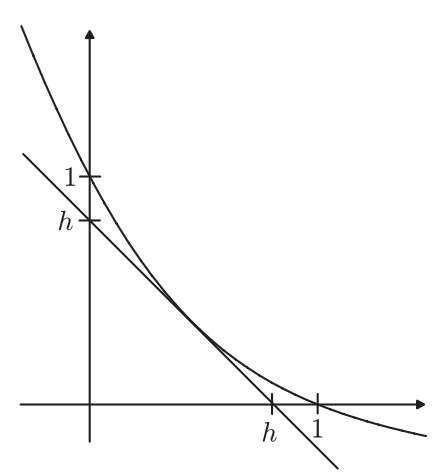

(a) The $\beta$-graph

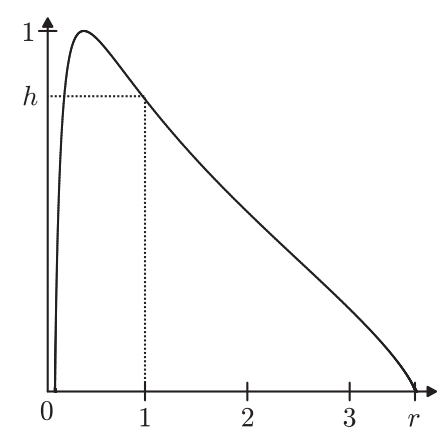

(b) The Lyapunov spectrum $s \mapsto \operatorname{dim}_{H}(\mathcal{L}(s))$

Fig. 2. The $\beta$-graph and the graph of the Lyapunov dimension spectrum in the Salem case for $\tau=0.08$; in both figures $h=0.8107 \ldots$ denotes the Hausdorff dimension of $\mathcal{D}_{\sim}$. The conjugacy $\Theta_{\tau}$ is $1 / r$-Hölder regular.

In order to compute $\operatorname{dim}_{H}\left(\mathcal{D}_{\sim}\left(S_{\tau}, T\right)\right)$, let $\nu_{\tau}$ be the $\left(p_{\tau}, 1-p_{\tau}\right)$-Bernoulli measure such $\int \psi d \nu_{\tau} / \int \varphi_{\tau} d \nu_{\tau}=1$. We then have

and hence

$$
1=\frac{\int \psi d \nu_{\tau}}{\int \varphi_{\tau} d \nu_{\tau}}=-p_{\tau} \log _{2} \tau-\left(1-p_{\tau}\right) \log _{2}(1-\tau)
$$

$$
p_{\tau}=\frac{1+\log _{2}(1-\tau)}{\log _{2} \tau-\log _{2}(1-\tau)} .
$$

One then immediately verifies that the supremum in Remark 1.6(2) is attained for $\mu=\nu_{\tau}$, and hence

$$
\operatorname{dim}_{\mathrm{H}}\left(\mathcal{D}_{\sim}\left(S_{\tau}, T\right)\right)=-p_{\tau} \log _{2} p_{\tau}-\left(1-p_{\tau}\right) \log _{2}\left(1-p_{\tau}\right) .
$$

The graphs of $\beta_{\tau}$ and of the corresponding dimension spectrum are given in Fig. 2. Also, Fig. 3(b) shows $\operatorname{dim}_{\mathrm{H}}\left(\mathcal{D}_{\sim}\left(S_{\tau}, T\right)\right)$ in dependence on $\tau$. 
Finally, let us mention that one can also explicitly calculate the number $s_{0}\left(S_{\tau}\right)$ which is determined by $\beta_{\tau}^{\prime}\left(s_{0}\left(S_{\tau}\right)\right)=-1$. A straightforward calculation gives

$$
s_{0}\left(S_{\tau}\right)=\left(\log \left(\tau^{-1}-1\right)\right)^{-1} \log \left(\frac{\log (2 \tau)}{\log (2 /(1-\tau))}\right) .
$$

ExAmple 2 (the sine family). Let $T$ be given as in the previous example, and for each $\tau \in(0,1)$ let the map $R_{\tau}: \mathcal{U} \rightarrow \mathcal{U}$ be defined by

$$
R_{\tau}(\xi):=2 \xi+\frac{\tau}{2 \pi} \sin (2 \pi \xi) \bmod 1 \text { for each } \xi \in \mathcal{U} \text {. }
$$

The associated conjugacies $\Psi_{\tau}$ are given by $\Psi_{\tau} \circ R_{\tau}=T \circ \Psi_{\tau}$ (see Fig. 4). We can then use Theorem 1.1 to compute the Hausdorff dimension of the set $\mathcal{D}_{\sim}\left(R_{\tau}, T\right)$ of points at which $\Psi_{\tau}$ is not differentiable in the generalised sense. This is plotted as a graph in Fig 3. (Note that taking the conjugacy in the other direction would yield exactly the same result.)

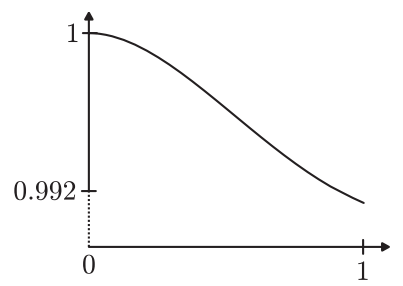

(a) The graph of $\tau \mapsto \operatorname{dim} D_{\sim}\left(R_{\tau}, T\right)$ for the sine family with parameter $\tau$

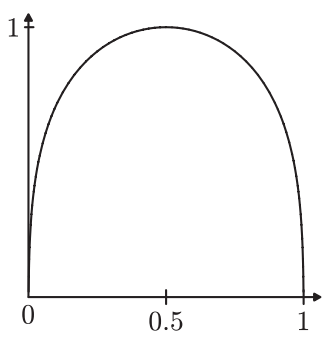

(b) The graph of $\tau \mapsto \operatorname{dim} D_{\sim}\left(S_{\tau}, T\right)$ for the Salem family with parameter $\tau$

Fig. 3. The two dimension spectra

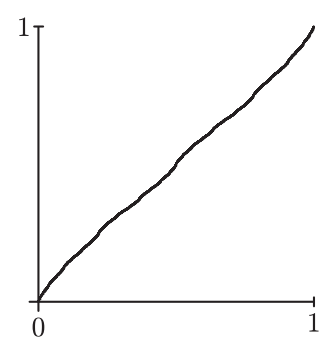

(a) $\tau=0.4$

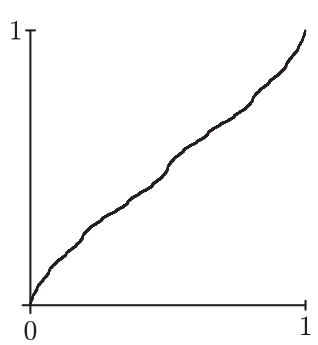

(b) $\tau=0.8$

Fig. 4. The graphs of the conjugating maps $\Psi_{\tau}$ for $\tau=0.4$ and $\tau=0.8$ in the sine-family example 


\section{Proofs of Propositions 1.4 and 1.5}

Proof of Proposition 1.4. We start by observing that the Hausdorff dimension of the set $\mathcal{D}_{\sim}$ depends regularly on the expanding maps. Let $T_{\tau}$ : $\mathcal{U} \rightarrow \mathcal{U}$ be elements of the Banach manifold of the $C^{k}$ family of expanding maps, with a $C^{k}$ dependence on $\tau \in(-\epsilon, \epsilon)$, say, and assume that $T_{0}$ is the usual $d$-to-1 linear expanding map. Let $0=a_{0}^{(\tau)}<a_{1}^{(\tau)}<\cdots<a_{d}^{(\tau)}=1$ denote the $T_{\tau}$-preimages of zero. For each $\alpha>0$, we then define the operator $\mathcal{I}_{\tau}: C^{\alpha}(\mathcal{U}, \mathbb{R}) \rightarrow C^{\alpha}(\mathcal{U}, \mathbb{R})$ on the space of $\alpha$-Hölder continuous functions (see e.g. [8]) by

$\left(\mathcal{T}_{\tau} h\right)(\xi):=\frac{1}{d} h\left(\left\{T_{\tau}(\xi)\right\}\right)+\frac{j}{d} \quad$ for each $\xi \in\left[a_{j}^{(\tau)}, a_{j+1}^{(\tau)}\right], j \in\{0, \ldots, d-1\}$.

Also, with $\|h\|_{\infty}$ denoting the usual supremum norm, we define a norm $\|\cdot\|$ on $C^{\alpha}(\mathcal{U}, \mathbb{R})$ by

$$
\|h\|:=\sup _{\xi \neq \eta} \frac{|h(\xi)-h(\eta)|}{|\xi-\eta|^{\alpha}}+\|h\|_{\infty} .
$$

We observe that on each of the intervals $\left[a_{j}^{(\tau)}, a_{j+1}^{(\tau)}\right]$ we have

$$
\left|\mathcal{T}_{\tau} h_{1}(\xi)-\mathcal{T}_{\tau} h_{2}(\xi)\right| \leq \frac{1}{d}\left|h_{1}\left(T_{\tau}(\xi)\right)-h_{2}\left(T_{\tau}(\xi)\right)\right| \leq \frac{1}{d}\left\|h_{1}-h_{2}\right\|_{\infty}
$$

and

$$
\begin{aligned}
\mid \mathcal{T}_{\tau}\left(h_{1}-h_{2}\right)(\xi) & -\mathcal{T}_{\tau}\left(h_{1}-h_{2}\right)(\eta)\left|\leq \frac{1}{d}\left\|h_{1}-h_{2}\right\|_{C^{\alpha}}\right| T_{\tau}(\xi)-\left.T_{\tau}(\eta)\right|^{\alpha} \\
& \leq \frac{1}{d}\left\|h_{1}-h_{2}\right\|_{C^{\alpha}}\left|T_{\tau}(\xi)-T_{\tau}(\eta)\right|^{\alpha} \\
& \leq\left(\frac{1}{d}\left\|h_{1}-h_{2}\right\|_{C^{\alpha}}\left\|T_{\tau}\right\|_{C^{1}}^{\alpha}\right)|\xi-\eta|^{\alpha} .
\end{aligned}
$$

In particular, for $\alpha>0$ sufficiently small, $\mathcal{T}_{\tau}$ is a contraction with respect to $\|\cdot\|$. Moreover, $I-\mathcal{T}_{\tau}: C^{\alpha}(\mathcal{U}, \mathbb{R}) \rightarrow C^{\alpha}(\mathcal{U}, \mathbb{R})$ is invertible, and by the Implicit Function Theorem there exists a $C^{k}$ family $\left\{h_{\tau} \in C^{\alpha}(\mathcal{U}, \mathcal{U}): \tau \in\right.$ $(-\epsilon, \epsilon)\}$ such that $h_{0}$ is the identity map and $\mathcal{I}_{\tau} h_{\tau}=h_{\tau}$.

Let us consider the map $H_{\tau}:(-\epsilon, \epsilon) \rightarrow C^{k-1}(\mathcal{U}) \times C^{\alpha}(\mathcal{U})$ given by $H_{\tau}(\tau):=\left(\log \left|T_{\tau}^{\prime}\right|, h_{\tau}\right)$. Clearly, it is $C^{k-1}$ as a map on Banach spaces. Also, we define the composition operator $\mathcal{O}: C^{k-1}(\mathcal{U}) \times C^{\alpha}(\mathcal{U}) \rightarrow C^{\alpha}(\mathcal{U})$ by $\mathcal{O}(f, g):=f \circ g$, which is $C^{k-2}$, by a result of [5]. We then consider the image of $H_{\tau}$ under $\mathcal{O}$, that is,

$$
\mathcal{O} \circ H_{\tau}: \tau \mapsto\left(\log \left|T_{\tau}^{\prime}\right|, h_{\tau}\right) \mapsto \mathcal{O}\left(\log \left|T_{\tau}^{\prime}\right|, h_{\tau}\right)=\log \left|T_{\tau}^{\prime}\right| \circ h_{\tau} \in C^{\alpha},
$$

which is again $C^{k-2}[5]$. (Note that if instead we would consider $\widetilde{\mathcal{O}}$ : $C^{k-1}(\mathcal{U}) \times C^{0}(\mathcal{U}) \rightarrow C^{0}(\mathcal{U})$, then $\widetilde{\mathcal{O}}\left(H_{\tau}\right)$ would be $C^{k-1}$; but we need to work with Hölder functions, which causes the loss of an extra derivative.) 
Now consider the potential function $\varphi_{\tau}: \xi \mapsto-\log \left|T_{\tau}^{\prime}\left(h_{\tau}\left(\pi_{T_{\tau}}(\xi)\right)\right)\right|$ defined on $\mathcal{U}$, and then let $\beta_{\tau}(s)$ be defined implicitly by

$$
P\left(-s \log \left|T_{\tau}^{\prime}\right|+\beta_{\tau}(s) \log \left|T_{0}^{\prime}\right|\right)=0 .
$$

Since the pressure function is analytic, the Implicit Function Theorem implies that the function $\tau \mapsto \beta_{\tau}$ is analytic. Also, it follows that $\tau \mapsto$ $\operatorname{dim}_{\mathrm{H}}\left(\mathcal{D}_{\sim}\left(T_{\tau}, T_{0}\right)\right)$ is a $C^{k-2}$ function (for an example see Fig. 3 ). This completes the proof of Proposition 1.4.

Proof of Proposition 1.5. The aim is to show that there exists a conjugacy between two elements of the space $C^{2}\left(\mathbb{S}^{1}\right)$ of $C^{2}$ expanding circle maps such that the Hausdorff dimension of the set of points at which this conjugacy is nondifferentiable in the generalised sense is arbitrarily close to 0 . We start by considering the Salem case but where the maps are defined on the circle $\mathbb{S}^{1}$. For ease of exposition, we use the same notation and let $T: \mathbb{S}^{1} \rightarrow \mathbb{S}^{1}$ and $S_{\tau}: \mathbb{S}^{1} \rightarrow \mathbb{S}^{1}$ refer to the circle maps which correspond to the interval maps defined in Example 1. The corresponding conjugacy $\Theta_{\tau}$ is given as before by $T \circ \Theta_{\tau}=\Theta_{\tau} \circ S_{\tau}$. From our analysis in Example 1 it is clear that $\operatorname{dim}_{\mathrm{H}}\left(\mathcal{D}_{\sim}\left(S_{\tau}, T\right)\right)$ tends to zero for $\tau$ tending to zero (see Fig. 2). However, whereas $T$ is a $C^{2}$ map of the circle, $S_{\tau}$ is clearly not (although it is always piecewise expanding $C^{2}$ when viewed as a map of $\mathcal{U}$ into itself). So, in order to find a $C^{2}$ example, we have to apply some perturbations to $S_{\tau}$. For this, let $\beta_{\tau}$ and $\psi, \varphi_{\tau}: \Sigma \rightarrow \mathbb{R}$ be given as in Example 1. As before we choose $s_{0}\left(S_{\tau}\right)$ satisfying $\beta_{\tau}^{\prime}\left(s_{0}\left(S_{\tau}\right)\right)=-1$. For the remaining part of the proof, let $\tau \in(0,1) \backslash\{1 / 2\}$ be fixed.

$C^{2}$ denseness. We use the metric $d_{\mathrm{KL}}$ considered by Keller and Liverani in [10]. This metric is given, for expanding piecewise $C^{2}$ maps $F$ and $G$ of the unit interval $\mathcal{U}$ into itself, by $d_{\mathrm{KL}}(F, G):=\inf \{\gamma>0 \mid \exists X \subseteq \mathcal{U} \exists$ a diffeomorphism $H: \mathcal{U} \rightarrow \mathcal{U}$ such that

$$
\begin{aligned}
& \lambda(X)>1-\gamma,\left.G\right|_{X}=\left.F \circ H\right|_{X} \text { and } \\
& \left.\forall \xi \in \mathcal{U}:|H(\xi)-\xi|<\gamma,\left|1-\left(H^{-1}\right)^{\prime}(H(\xi))\right|<\gamma\right\} .
\end{aligned}
$$

One immediately verifies that there exists a sequence $\left(S^{(n)}\right)_{n \in \mathbb{N}}$ of functions in $C^{2}\left(\mathbb{S}^{1}\right)$ such that $\lim _{n \rightarrow \infty} d_{\mathrm{KL}}\left(S^{(n)}, S_{\tau}\right)=0$, where the $S^{(n)}$ are viewed as interval maps.

Norms and operators. Let $B_{0}(\mathcal{U}):=\left\{f: \mathcal{U} \rightarrow \mathbb{R} \mid\|f\|_{0}<\infty\right\}$ be the Banach space with the combined norm $\|\cdot\|_{0}$ given by $\|f\|_{0}:=\|f\|_{1}+$ $\|f\|_{B V}$, where $\|\cdot\|_{1}$ denotes the $L^{1}$ norm and $\|\cdot\|_{B V}$ the bounded variation seminorm, given by $\sup \left\{\sum_{i=1}^{n}\left|f\left(\xi_{i+1}\right)-f\left(\xi_{i}\right)\right| \mid 0 \leq x_{1}<\cdots<x_{n} \leq 1\right.$, $n \in \mathbb{N}\}$. Also, let the weak operator norm $\|\cdot\|_{W}$ be given by $\|\mathcal{L}\|_{W}:=$ $\sup \left\{\|\mathcal{L}(g)\|_{1} \mid g \in B_{0}(\mathcal{U}),\|g\|_{B V} \leq 1\right\}$. Finally, for an expanding map $S: \mathcal{U} \rightarrow \mathcal{U}$ we define the transfer operator $\mathcal{L}_{S}: B_{0}(\mathcal{U}) \rightarrow B_{0}(\mathcal{U})$, for 
$g \in B_{0}(\mathcal{U})$ and $\xi \in \mathcal{U}$, by

$$
\mathcal{L}_{S} g(\xi)=\sum_{S(\eta)=\xi}\left|S^{\prime}(\eta)\right|^{-1} g(\eta) .
$$

Continuity. Firstly, note that it can be shown that $\lim _{n \rightarrow \infty} \| \mathcal{L}_{S^{(n)}}-$ $\mathcal{L}_{S_{\tau}} \|_{W}=0$ (see comment (a) on page 143 of [10]). Furthermore, by [10, Corollary 1], for each $t \in \mathbb{R}$ fixed, the leading eigenvalues of the operators $\mathcal{L}_{S^{(n)}}$ converge to the leading eigenvalue of $\mathcal{L}_{S_{\tau}}$. That is,

$$
\lim _{n \rightarrow \infty} P\left(t \log \left|\left(S^{(n)}\right)^{\prime}\right|\right)=P\left(t \log \left|S_{\tau}^{\prime}\right|\right) .
$$

Local uniform convergence. Recall that the map given by

$$
t \mapsto \beta_{S^{(n)}}(t):=P\left(-t \log \left(S^{(n)}\right)^{\prime}\right) / \log 2
$$

is differentiable and convex. Using the above 'Continuity', we then have $\lim _{n \rightarrow \infty} \beta_{S^{(n)}}(t)=\beta_{S_{\tau}}(t)$ for each $t \in \mathbb{R}$ fixed. Since pointwise convergence of sequences of differentiable convex functions implies local uniform convergence (see [19, Theorem 10.8]), we now conclude that

$$
\lim _{n \rightarrow \infty} \beta_{S^{(n)}}\left(s_{0}\left(S^{(n)}\right)\right)+s_{0}\left(S^{(n)}\right)=\beta_{S_{\tau}}\left(s_{0}\left(S_{\tau}\right)\right)+s_{0}\left(S_{\tau}\right) .
$$

Since $\beta_{S}\left(s_{0}(S)\right)+s_{0}(S)=\operatorname{dim}_{\mathrm{H}}\left(\mathcal{D}_{\sim}(S, T)\right)$, this finishes the proof of the proposition.

Acknowledgements. Some of the work for this paper was undertaken during workshops at the Banach Centre (Warsaw) and the Schrödinger Institute (Vienna), and the authors would like to thank these institutions for their hospitality.

\section{References}

[1] V. Baladi, Positive Transfer Operators and Decay of Correlations, Adv. Ser. Nonlinear Dynam. 16, World Sci., River Edge, NJ, 2000.

[2] R. Bowen, Hausdorff dimension of quasi-circles, Publ. Math. IHES 50 (1979), 11-25.

[3] G. Cui, On the smoothness of conjugacy for circle covering maps, Acta Math. Sinica (N.S.) 12 (1996), 122-125.

[4] R. Darst, The Hausdorff dimension of the nondifferentiability set of the Cantor function is $[\ln 2 / \ln 3]^{2}$, Proc. Amer. Math. Soc. 119 (1993), 105-108.

[5] R. de la Llave and R. Obaya, Regularity of the composition operator in spaces of Hölder functions, Discrete Contin. Dynam. Systems 5 (1999), 157-184.

[6] M. Denker and W. Philipp, Approximation by Brownian motion for Gibbs measures and flows under a function, Ergodic Theory Dynam. Systems 4 (1984), 541-552.

[7] K. J. Falconer, One-sided multifractal analysis and points of nondifferentiability of devil's staircases, Math. Proc. Cambridge Philos. Soc. 136 (2004), 67-174.

[8] B. Hasselblatt and A. Katok, Introduction to the Modern Theory of Dynamical Systems, Encyclopedia Math. Appl. 54, Cambridge Univ. Press, Cambridge, 1995. 
[9] Y. Jiang, Renormalization and Geometry in One-Dimensional and Complex Dynamics, Adv. Ser. Nonlinear Dynam. 10, World Sci., 1996.

[10] G. Keller and C. Liverani, Stability of the spectrum for transfer operators, Ann. Scuola Norm. Sup. Pisa Cl. Sci. (4) 28 (1999), 141-152

[11] M. Kesseböhmer and B. O. Stratmann, Hölder-differentiability of Gibbs distribution functions, Math. Proc. Cambridge Philos. Soc. 147 (2009), 489-503.

[12] - - - Fractal analysis for sets of nondifferentiability of Minkowski's question mark function, J. Number Theory 128 (2008), 2663-2686.

[13] - - - A multifractal analysis for Stern-Brocot intervals, continued fractions and Diophantine growth rates, J. Reine Angew. Math. 605 (2007), 133-163.

[14] A. Manning, A relation between Lyapunov exponents, Hausdorff dimension and entropy, Ergodic Theory Dynam. Systems 1 (1981), 451-459.

[15] H. Minkowski, Zur Geometrie der Zahlen, in: Verhandlungen des III. internationalen Mathematiker-Kongresses in Heidelberg, 1904, 164-173. Also in: Gesammelte Abhandlungen, Vol. 2, Teubner, 1911, 43-52.

[16] G. D. Mostow, Strong rigidity of locally symmetric spaces, Ann. of Math. Stud. 78, Princeton Univ. Press, 1972.

[17] Ya. B. Pesin, Dimension Theory in Dynamical Systems. Contemporary Views and Applications, Chicago Lectures in Math., Univ. of Chicago Press, Chicago, IL, 1997.

[18] F. Przytycki and M. Urbański, Conformal Fractals - Ergodic Theory Methods, Cambridge Univ. Press, to appear.

[19] R. T. Rockafellar, Convex Analysis, Princeton Math. Ser. 28, Princeton, NJ, 1970.

[20] R. Salem, On some singular monotonic functions which are strictly increasing, Trans. Amer. Math. Soc. 53 (1943), 427-439.

[21] M. Shub and D. Sullivan, Expanding endomorphisms of the circle revisited, Ergodic Theory Dynam. Systems 5 (1985), 285-289.

[22] D. Sullivan, Quasiconformal homeomorphisms in dynamics, topology, and geometry, in: Proc. Int. Congress Math., Vol. 1, 2 (Berkeley, CA 1986), Amer. Math. Soc., Providence, RI, 1987, 1216-1228.

[23] L.-S. Young, Dimension, entropy and Lyapunov exponents. Ergodic Theory Dynam. Systems 2 (1982), 109-124.

Thomas Jordan

Department of Mathematics

University of Bristol

Bristol, BS8 1TW, UK

E-mail: thomas.jordan@bristol.ac.uk

Mark Pollicott

Mathematics Institute

University of Warwick

Coventry, CV4 7AL, UK

E-mail: mpollic@maths.warwick.ac.uk
Marc Kesseböhmer Fachbereich 3-Mathematik und Informatik Universität Bremen D-28359 Bremen, Germany E-mail: mhk@math.uni-bremen.de

Bernd O. Stratmann Mathematics Institute University of St Andrews St Andrews, KY16 9SS, Scotland E-mail: bos@maths.st-and.ac.uk

Received 29 January 2009;

in revised form 26 June 2009 